\title{
Light-scattering signal may indicate critical time zone to rescue brain tissue after hypoxia
}

\author{
Satoko Kawauchi, ${ }^{a}$ Shunichi Sato, ${ }^{\mathrm{b}}$ Yoichi Uozumi, ${ }^{\mathrm{c}}$ Hiroshi Nawashiro, ${ }^{\mathrm{c}}$ Miya Ishihara, ${ }^{\mathrm{a}}$ and Makoto Kikuchi ${ }^{\mathrm{a}}$ \\ ${ }^{a}$ National Defense Medical College, Department of Medical Engineering, 3-2 Namiki, Tokorozawa, Saitama 359-8513, Japan \\ ${ }^{b}$ National Defense Medical College Research Institute, Division of Biomedical Information Sciences, 3-2 Namiki, \\ Tokorozawa, Saitama 359-8513, Japan \\ c National Defense Medical College, Department of Neurosurgery, 3-2 Namiki, Tokorozawa, Saitama 359-8513, Japan
}

\begin{abstract}
A light-scattering signal, which is sensitive to cellular/subcellular structural integrity, is a potential indicator of brain tissue viability because metabolic energy is used in part to maintain the structure of cells. We previously observed a unique triphasic scattering change (TSC) at a certain time after oxygen/glucose deprivation for blood-free rat brains; TSC almost coincided with the cerebral adenosine triphosphate (ATP) depletion. We examine whether such TSC can be observed in the presence of blood in vivo, for which transcranial diffuse reflectance measurement is performed for rat brains during hypoxia induced by nitrogen gas inhalation. At a certain time after hypoxia, diffuse reflectance intensity in the near-infrared region changes in three phases, which is shown by spectroscopic analysis to be due to scattering change in the tissue. During hypoxia, rats are reoxygenated at various time points. When the oxygen supply is started before TSC, all rats survive, whereas no rats survive when the oxygen supply is started after TSC. Survival is probabilistic when the oxygen supply is started during TSC, indicating that the period of TSC can be regarded as a critical time zone for rescuing the brain. The results demonstrate that light scattering signal can be an indicator of brain tissue reversibility. $\odot 2011$ Society of Photo-Optical Instrumentation Engineers (SPIE). [DOI: 10.1117/1.3542046]
\end{abstract}

Keywords: light scattering; brain; tissue viability; anoxic depolarization; multiwavelength diffuse reflectance measurement.

Paper 10400PR received Jul. 14, 2010; revised manuscript received Dec. 8, 2010; accepted for publication Dec. 27, 2010; published online Feb. 17, 2011.

\section{Introduction}

Brain tissue is highly vulnerable to ischemia and hypoxia; after onsets of these events, the brain becomes functionally silent, but there still remains a time period that allows effective treatment to save brain tissue (e.g., blood reperfusion). ${ }^{1}$ However, there is no established modality to discern this critical time period. Thus, a method for noninvasive, real-time monitoring of tissue viability in the brain has been strongly desired.

Viability of brain tissue relates not only to energy metabolism but also to the structural integrity of cells and organelles in the tissue. ${ }^{1}$ Thus, light scattering, which is sensitive to morphological changes of cells and organelles, ${ }^{2}$ can be a potential indicator of tissue viability in the brain. In the near-infrared (NIR) spectral region (650-1000 nm), the reduced scattering coefficient of gray matter is two orders of magnitude larger than the absorption coefficient. ${ }^{3}$ Thus, the use of light scattering enables sensitive monitoring of tissue viability in brains.

We previously performed simultaneous measurements of light scattering and absorption due to redox changes of cytochrome c oxidase $(\mathrm{CcO})$, which is the terminal electron acceptor in the mitochondrial respiratory chain, ${ }^{4}$ for blood-free rat brains after oxygen and glucose deprivation. ${ }^{5}$ We observed a unique triphasic change in light scattering at a certain period of time after starting oxygen and glucose deprivation, after which light scattering remained at a high level. Observation of the

Address all correspondence to: Satoko Kawauchi, National Defense Medical College, Department of Medical Engineering, 3-2 Namiki, Tokorozawa, Saitama 359-8513, Japan. Tel: 81-4-2995-1627; Fax: 81-4-2996-5199; E-mail: skawauch@ndmc.ac.jp tissue by transmission electron microscopy suggested that the increased light scattering after the triphasic scattering change (TSC) is due to deformed or enlarged mitochondria and expanded dendrites. The TSC was preceded by reduction of heme $\mathrm{aa}_{3}$ in $\mathrm{CcO}$, and reduction of $\mathrm{CuA}$ in $\mathrm{CcO}$ started during the TSC. Direct measurement of cerebral ATP concentration showed a significant decrease in ATP during TSC (i.e., the start of TSC preceded the ATP exhaustion). ${ }^{6}$ Direct current (DC) potential was also measured for the rat brains, and the measurements suggested that TSC is associated with anoxic depolarization (AD). Because $\mathrm{AD}$ accompanies drastic movements of ions across the cellular membrane, ${ }^{7,8}$ cellular/subcellular morphological alterations would occur, resulting in change in the light scattering for the tissue. ${ }^{8-10} \mathrm{AD}$ is known to be a critical event related to cell survival; if energy is restored in the early phase of $\mathrm{AD}$, neuronal function can recover, while long-lasting $\mathrm{AD}$ causes irreversible tissue damage. 8,11

In the present study, we first examined whether TSC can be observed in the presence of blood in vivo, for which diffuse reflectance was measured for rat brains during hypoxia induced by nitrogen gas inhalation. The measurements showed that at a certain period of time after starting hypoxia, the diffuse reflectance in the NIR region changed in three phases; the change was similar to that observed for blood-free rat brains after oxygen and glucose deprivation. To verify whether this triphasic reflectance change was due to change in light scattering, we examined the wavelength dependence of the reflectance intensity changes. We also performed an experiment to reoxygenate rat brains after hypoxia at various time points under spontaneous

1083-3668/2011/16(2)/027002/8/\$25.00 (C) 2011 SPIE 
respiration, and the results revealed the dependence of the rat recovery rate on reoxygenation timing in terms of the phases of triphasic reflectance change.

\section{Materials and Methods}

All experiments were carried out in accordance with the Guide for Laboratory Animal Facilities and Care Regulation of the National Defense Medical College, Saitama, Japan. Figure 1 shows a diagram of the experimental setup for diffuse reflectance measurement. Sprague-Dawley male rats weighing 200-280 g were anesthetized with pentobarbital sodium $(50 \mathrm{mg} / \mathrm{kg}$ animal weight) and placed in a stereotactic frame. A pair of $800-\mu \mathrm{m}$ core-diameter optical fibers with center-to-center separation of $2 \mathrm{~mm}$ was placed on the exposed parietal bone of the right hemisphere. The fiber tips were in direct contact with the skull, which was not thinned. As light sources, we employed either a combination of red and green light-emitting diodes (LEDs) and NIR laser diode (LD) ${ }^{5,6}$ or a tungsten lamp (BPS120, B \& W Tek, Inc., Newark, Delaware) with a long-wavelength-absorbing filter, which emits broadband light from 400 to $1000 \mathrm{~nm}$; the latter was used for spectral analysis of diffuse reflectances. The irradiation powers of the red LED, green LED, NIR LD, and lamp with a filter were set at $10,25,1.8$, and $350 \mu \mathrm{W}$, respectively.

Transcranial diffuse reflectance measurements were performed for rat brains during hypoxia that was induced by $\mathrm{N}_{2}$ gas inhalation through a face mask under spontaneous respiration. The body temperature was kept constant at $32.0 \pm 0.5^{\circ} \mathrm{C}$ by using a temperature-controlled body mat. After starting $\mathrm{N}_{2}$ inhalation, heart beat was observed for $\sim 470 \mathrm{~s}$ without reoxygenation. Before and during optical measurements, arterial oxygen saturation $\left(\mathrm{SpO}_{2}\right)$ was monitored with a pulse oximeter (8600V, Nonin Medical, Inc., Plymouth, Minnesota) that was attached to the forelimb, and electroencephalogram (EEG) was recorded using an amplifier (ER-1, Cygnus Technology, Inc., Southport, North Carolina) with steel electrodes screwed in the frontal and parietal bones of the contralateral (left) hemisphere. Pupil diameter was also measured for randomly selected rats to examine the time-dependent change in brain stem function.

Diffuse reflectance intensities were measured with a polychromater (PMA-11, Hamamatsu Photonics K.K., Hamamatsu, Japan) from the visible to NIR spectral region. In the NIR region, light scattering is a dominant attenuation mechanism, and change in light scattering can therefore be monitored by measuring change in reflectance intensity. To confirm this, we assessed wavelength dependence of the reflectance intensity changes in the spectral region from visible to NIR. Time courses of NIR reflectance intensities were measured at 730,805 , and $830 \mathrm{~nm}$;

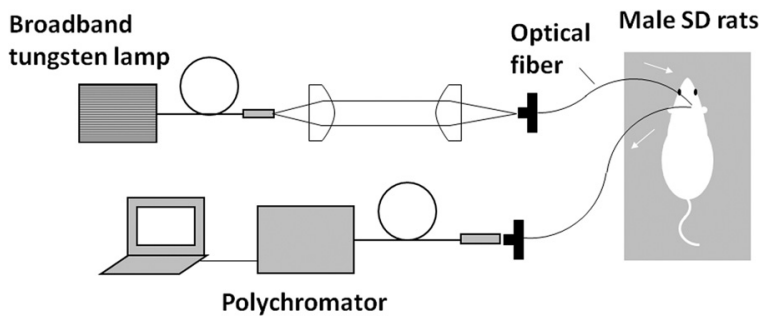

Fig. 1 Experimental setup for diffuse reflectance measurement for rat brains.
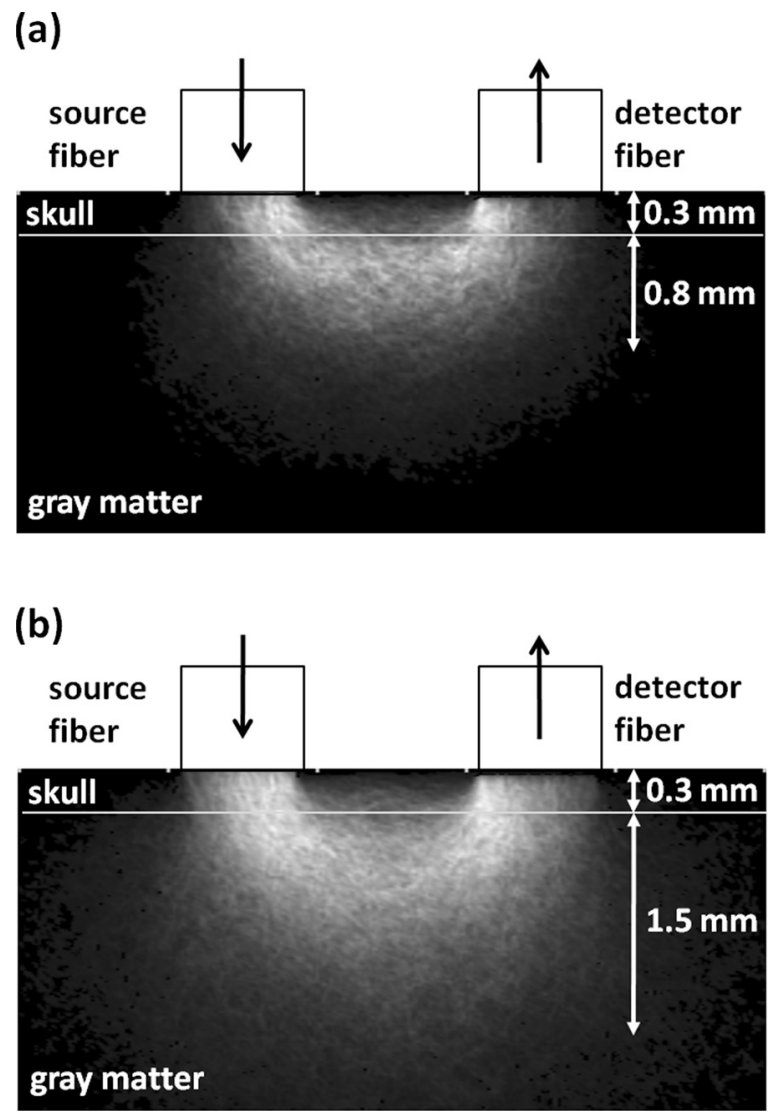

Fig. 2 Optical paths of (a) diffuse light reflectances at $560 \mathrm{~nm}$ and (b) those at $805 \mathrm{~nm}$ in cross-sectional views by Monte Carlo simulation. The source and detector fibers were $800 \mu \mathrm{m}$ in core diameter, and they were separated by $2 \mathrm{~mm}$. The maximum optical penetration depths were calculated to be $\sim 0.8 \mathrm{~mm}$ at $560 \mathrm{~nm}$ and $\sim 1.5 \mathrm{~mm}$ at $805 \mathrm{~nm}$ in the gray matter (cerebral cortex).

$805 \mathrm{~nm}$ is an isosbestic point of oxy-Hb and deoxy-Hb, and 730 and $830 \mathrm{~nm}$ lie in the shorter wavelength side and in the longer wavelength side of the isosbestic point, respectively. ${ }^{12}$ For monitoring absorption signals related to hemodymanics in the brain, diffuse reflectance intensities at $569 \mathrm{~nm}\left(\mathrm{R}_{569}\right)$ and $560 \mathrm{~nm}\left(\mathrm{R}_{560}\right)$ were measured; $569 \mathrm{~nm}$ is also an isosbestic point of oxy-Hb and deoxy-Hb, ${ }^{12}$ and $\mathrm{R}_{569}$ therefore reflects the total hemoglobin (total $\mathrm{Hb}$ ) concentration. To monitor relative change in deoxy-Hb concentration, $\mathrm{R}_{560}$ was normalized by $\mathrm{R}_{569}$; the absorption coefficient of deoxy-Hb is higher than that of oxy-Hb at $560 \mathrm{~nm}^{12}$

Monte Carlo simulation was performed to estimate the detectable tissue region with the present fiber configuration, for which optical properties reported in the literature were used. ${ }^{13,14}$ Because optical properties of the skull at wavelengths of $<650 \mathrm{~nm}$ could not be determined, we used the values obtained by extrapolating published data ${ }^{14}$ (e.g., $\mu_{\mathrm{a}}=4.5 \mathrm{~cm}^{-1}$, $\mu_{\mathrm{s}}=388 \mathrm{~cm}^{-1}$, and $g=0.91$ at $\left.560 \mathrm{~nm}\right)$. The simulation showed that maximum depths of the main measurement regions were $\sim 0.8 \mathrm{~mm}$ at $560 \mathrm{~nm}$ and $\sim 1.5 \mathrm{~mm}$ at $805 \mathrm{~nm}$ from the surface of the gray matter (Fig. 2). This suggests that the measured optical signals came from cortical layers I-IV at 560 and 569 $\mathrm{nm}$ and cortical layers I-VI at $805 \mathrm{~nm}$.

To examine the recovery rates of rats by reoxygenation after starting hypoxia, inhalation gas was switched from $\mathrm{N}_{2}$ to $\mathrm{O}_{2}$ at 
different time points under spontaneous respiration. For rats that survived, weight and body movement were recorded for up to two weeks after reoxygenation.

\section{Results and Discussion}

Figure 3(a) shows typical time courses of normalized diffuse reflectance intensities at 730,805 , and $830 \mathrm{~nm}$ during hypoxia induced by $\mathrm{N}_{2}$ gas inhalation in a rat; each curve shows the integrated reflectance intensity for the wavelength of interest $\pm 5 \mathrm{~nm}$ to increase the signal-to-noise ratio. Immediately after starting $\mathrm{N}_{2}$ inhalation, reflectance intensities at 805 and $830 \mathrm{~nm}$ showed a small increase and a gradual decrease and then showed a triphasic change: increase (phase I), decrease (phase II), and increase (phase III) from $\sim 130$ to $\sim 210 \mathrm{~s}$; thereafter, they slightly increased by $\sim 500 \mathrm{~s}$ and remained at a high level until the end of the measurement ( $\sim 940 \mathrm{~s})$. Although the level of reflectance intensity at $730 \mathrm{~nm}$ was lower, the intensity showed a similar temporal behavior to those at 805 and $830 \mathrm{~nm}$. Time courses of $\mathrm{SpO}_{2}$ and EEG activity that were simultaneously measured with the reflectance intensities are shown in Figs. 3(b) and 3(c), respectively. After starting $\mathrm{N}_{2}$ inhalation, $\mathrm{SpO}_{2}$ rapidly decreased and reached $\sim 20 \%$ at $\sim 60 \mathrm{~s}$. Even after this rapid $\mathrm{SpO}_{2}$ decrease, EEG still showed a normal pattern for $\sim 30 \mathrm{~s}$, and it became a flat line pattern at $\sim 50 \mathrm{~s}$, indicating cessation of electrophysiological activity in the brain. $\mathrm{SpO}_{2}$ could not be detected due to a weakened arterial pulse from $210 \mathrm{~s}$ after starting $\mathrm{N}_{2}$ gas inhalation. Intrinsic absorption signals related to hemodynamics [i.e., reflectance intensity at $569 \mathrm{~nm}\left(\mathrm{R}_{569}\right)$ and reflectance intensity at $560 \mathrm{~nm}$ normalized by that at $\left.569 \mathrm{~nm}\left(\mathrm{R}_{569} / \mathrm{R}_{560}\right)\right]$ are also shown in Figs. 3(d) and 3(e), respectively; $R_{569}$ reflects total $\mathrm{Hb}$ concentration and $\mathrm{R}_{560} / \mathrm{R}_{569}$ indicates relative change in deoxy-Hb concentration. After starting $\mathrm{N}_{2}$ inhalation, $\mathrm{R}_{560} / \mathrm{R}_{569}$ showed a rapid decrease (rapid $\mathrm{Hb}$ deoxygenation) for $\sim 30 \mathrm{~s}$, after which $\mathrm{R}_{569}$ showed that total $\mathrm{Hb}$ concentration [i.e., cerebral blood volume (CBV)], started to increase and then decreased at $\sim 30 \mathrm{~s}$ [Fig. 3(d)]. These CBV changes show reactive hyperemia followed by hypoperfusion.

The wavelength-dependent differences in the NIR reflectance intensity levels [Fig. 3(a)] are attributable to different absorption levels caused by $\mathrm{Hb}$ deoxygenation. Small peaks observed at $\sim 30 \mathrm{~s}$ at all wavelengths $(730,805$, and $830 \mathrm{~nm})$ are thought to reflect changes in light scattering, because the wavelength dependence of the reflectance intensity changes was small in this period (data not shown). Because this scattering change appeared after the rapid $\mathrm{Hb}$ deoxygenation [Fig. 3(e)] and coincided with the onset of large increase in CBV [Fig. 3(d)], the scattering change would be caused by cellular/vascular responses to the rapid tissue hypoxia. The triphasic changes in NIR reflectance intensities [Fig. 3(a)] started $\sim 100 \mathrm{~s}$ after the $\mathrm{Hb}$ deoxygenation [Fig. 3(e)] and $\sim 80 \mathrm{~s}$ after the cessation of EEG activity [Fig. 3(c)], and the changes occurred almost synchronously with the CBV decrease (hypoperfusion) after the CBV increase (hyperemia) [Fig. 3(d)]. After the triphasic reflectance changes, reflectance intensities in the NIR region slightly increased by $\sim 500 \mathrm{~s}$ and remained at a high level until the end of the measurement ( $\sim 940 \mathrm{~s})$. After the triphasic reflectance change, reflectance intensity at $569 \mathrm{~nm}$ indicated gradual CBV decrease due to cardiac arrest. The same measurements were performed for 11 other rats, and the charac-
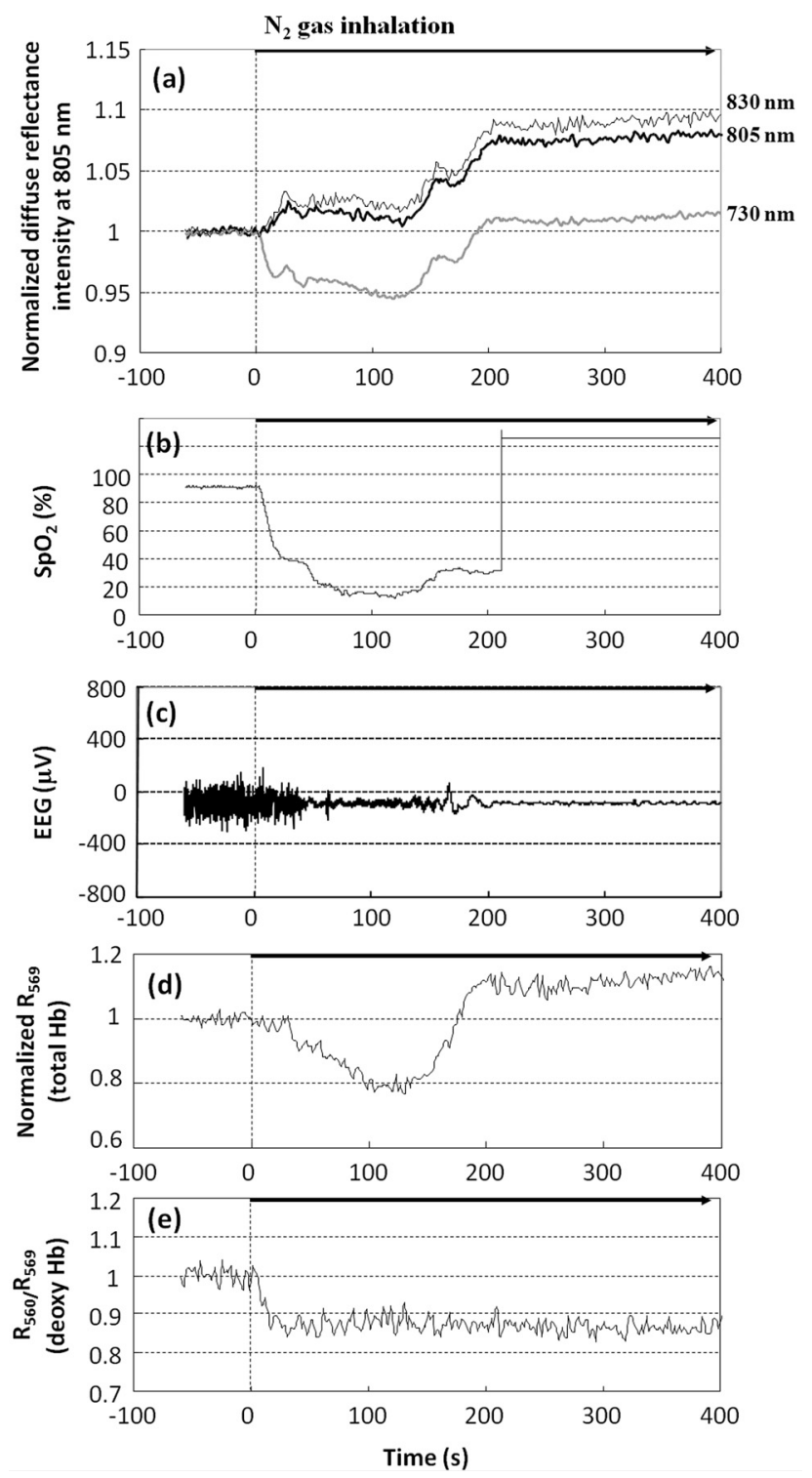

Fig. 3 Time courses of (a) normalized diffuse reflectance intensities at 730, 805, and $830 \mathrm{~nm}$, (b) arterial oxygen saturation, (c) EEG activity, (d) normalized diffuse reflectance intensity at $569 \mathrm{~nm}\left(\mathrm{R}_{569}\right)$, indicating relative change in total $\mathrm{Hb}$ concentration, and (e) normalized diffuse reflectance intensity at $560 \mathrm{~nm}\left(R_{560}\right)$ divided by $R_{569}$, indicating relative change in deoxy-Hb concentration. Nitrogen gas inhalation was started at $t=0$. Reflectance intensities at 730, 805, and $830 \mathrm{~nm}$ in (a) showed a triphasic change: increase (phase I), decrease (phase II), and increase (phase III) from $\sim 130$ to $\sim 210 \mathrm{~s}$.

teristics of sequential temporal changes in the NIR reflectance intensities, $\mathrm{SpO}_{2}$, EEG, and hemodynamics-related optical signals were highly reproducible. The starting time of the triphasic reflectance change was $128 \pm 14 \mathrm{~s}$, and its duration was $64 \pm 10 \mathrm{~s}$. The averaged reflectance changes in the three phases were $0.030 \pm 0.0058$ (phase I), $-0.0079 \pm 0.0061$ (phase II), and $0.042 \pm 0.011$ (phase III); after phase III, change in reflectance intensity remained small until the end of the measurements ( $940 \mathrm{~s}$ ). During $\mathrm{N}_{2}$ inhalation, pupil diameters of rats increased almost coincidentally with the triphasic reflectance change (data not shown), indicating that impairment 
of brain stem function almost coincided with the triphasic reflectance change.

To examine the origin of the triphasic changes in NIR reflectance intensities, we performed spectral analysis of the reflectance intensity changes in phases I, II, and III, shown in Fig. 3(a). Figure 4 shows the wavelength dependence of the reflectance intensity change rate in each phase, which was derived by dividing the amount of reflectance intensity change in each phase by the time duration of the phase. The level of reflectance intensity change rate at a specific wavelength reflects the contribution of a chromophore and/or

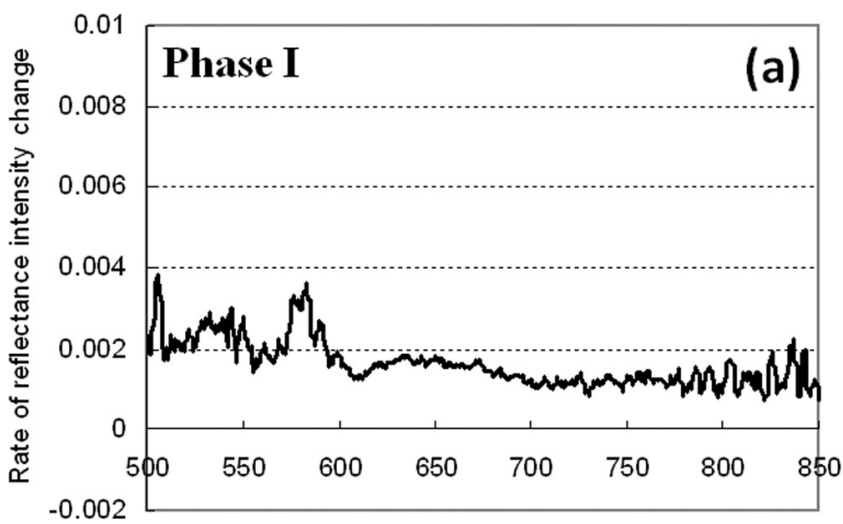

Wavelength $(\mathrm{nm})$

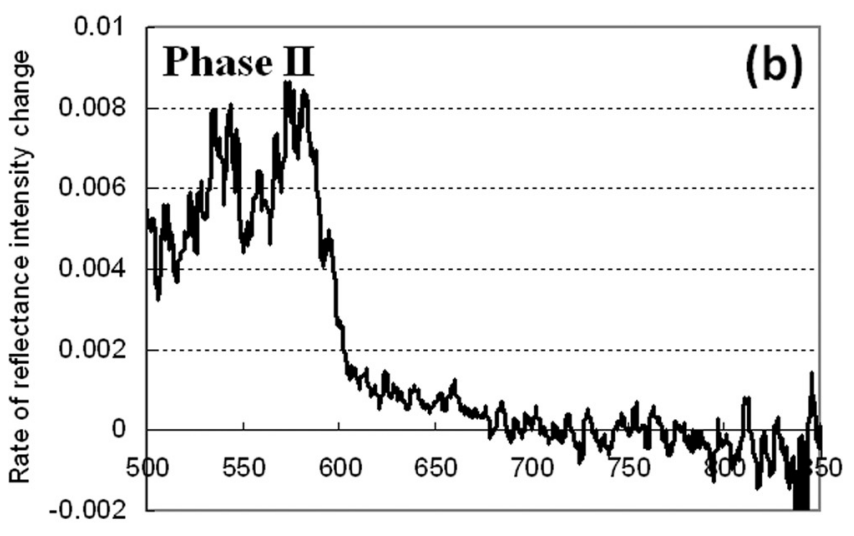

Wavelength $(\mathrm{nm})$

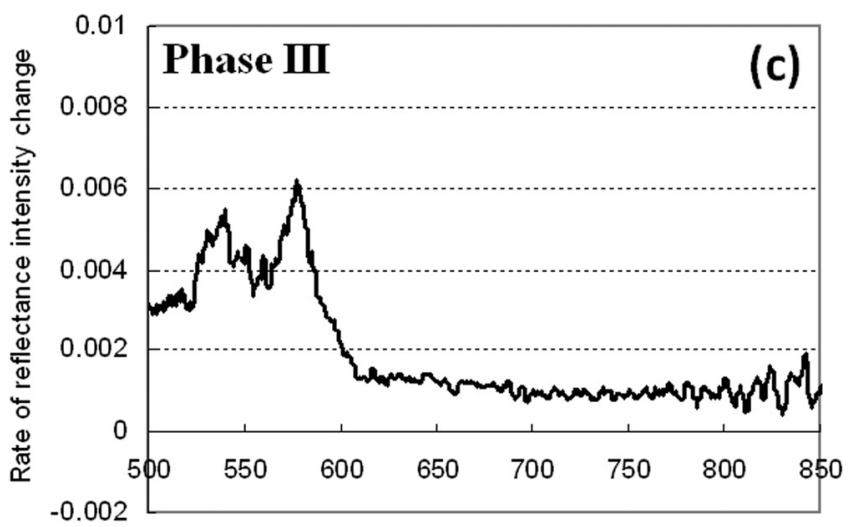

Wavelength $(\mathrm{nm})$

Fig. 4 Wavelength dependence of the reflectance intensity change rate in each phase of the triphasic reflectance change shown in Fig. 3(a): (a) phase I, (b) phase II, and (c) phase III. scattering to the temporal reflectance intensity change at the wavelength. The reflectance intensity change rates in the region of 500-600 $\mathrm{nm}$ should dominantly reflect change in $\mathrm{Hb}$ concentration, while a flat wavelength dependence of the reflectance intensity change rate in the NIR region $(650-900 \mathrm{~nm})$ indicates the contribution of light scattering change. ${ }^{2,3}$ In phase I [Fig. 4(a)], the wavelength dependence was relatively small over the whole spectral region. In phases II and III [Figs. 4(b) and 4(c)], the reflectance intensity change rates in the region of 500-600 $\mathrm{nm}$ showed large wavelength dependence with two major peaks, reflecting decreased $\mathrm{Hb}$ concentrations. In all phases, the wavelength dependence of the reflectance intensity change rates was small in the NIR region, indicating that the triphasic reflectance changes originated from light-scattering change. The wavelength analysis was performed for 11 other rats. In the region of $500-600 \mathrm{~nm}$, we observed some differences in the wavelength dependence of the reflectance intensity change rates between animals. In one rat, the effect of decreased $\mathrm{Hb}$ concentration was distinct even in phase I as shown in Figs. 4(b) and 4(c). In three rats, the effect of $\mathrm{Hb}$ decrease was small not only in phase I but also in phase II, as shown in Fig. 4(a). In some other cases, we observed a single-peaked wavelength dependence in the region of 500-600 $\mathrm{nm}$. These different behaviors indicate different $\mathrm{Hb}$ oxygenation/deoxygenation levels between animals during the triphasic NIR reflectance change. Despite these variations observed in the visible spectral region, the wavelength dependence of the reflectance intensity change rates in the NIR region was small in all rats investigated. These results demonstrate that TSC observed in the brains of rat saline-perfusion models is observable also in brains of in vivo rat $\mathrm{N}_{2}$ inhalation models by using NIR light. Hereafter, the triphasic NIR reflectance change observed in the present $\mathrm{N}_{2}$ inhalation model is also referred to as TSC.

TSCs observed during hypoxia in the present animal model should also be associated with $\mathrm{AD},{ }^{7,8}$ as in our previous bloodfree rat brain model during oxygen-glucose deprivation. ${ }^{5,6} \mathrm{We}$ speculated in our previous study that the initial scattering increase is due to drastic movements of ions across the cellular membrane accompanied by AD (phase I) and that the subsequent scattering decrease is due to restoration of ions (phase II). Because this process requires much energy, ${ }^{15} \mathrm{Na}^{+} / \mathrm{K}^{+}$ATPase is impaired and consequently movements of ions occur again, causing scattering increase in phase III. ${ }^{5,6}$ On the basis of this speculation, we assumed that cells in the brain can recover if oxygen is restored by phase II due to the preserved ion pump. In phase III and after TSC, on the other hand, oxygen restoration does not work and cells would be irreversible due to membrane failure.

Figure 5 shows recovery (survival) rates of rats that were reoxygenated after hypoxia. Reoxygenation was started at different time points: before, during, and after TSCs. When reoxygenation was started before TSC, all rats survived $(n=8)$, whereas all rats died $(n=8)$ when reoxygenation was started after TSC. With reoxygenation started in phases I, II, and III of TSC, survival rates were $43 \%(n=14), 10 \%(n=10)$, and $20 \%(n=10)$, respectively. Unexpectedly, there was no apparent phase-dependent tendency in survival rates. The rats that survived did not show any disability of motor function during the two-week follow-up period after reoxygenation. 


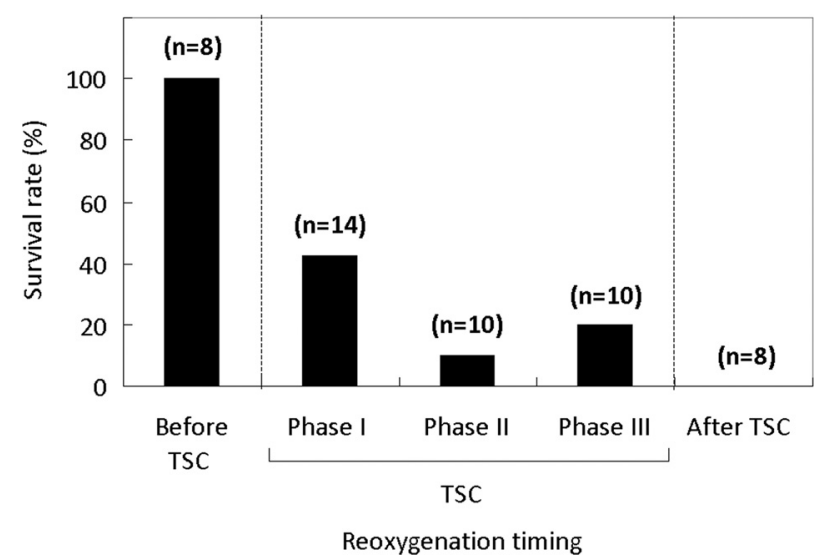

Fig. 5 Recovery (survival) rates of rats as a function of time span in which oxygen supply was started after hypoxia. Phases I, II, and III correspond to each phase of the TSC.

Figure 6 shows typical time courses of the same signals as those shown in Fig. 3 (except $R_{730}$ and $R_{830}$ ) for a rat that was reoxygenated before TSC. Figure 7 shows typical time courses of signals for a rat that was reoxygenated during TSC and survived, and Fig. 8 shows typical time courses of signals for a rat that was reoxygenated during TSC but died. When reoxygenation was started before TSC (Fig. 6), hemodynamics-related absorption signals showed that $\mathrm{CBV}$ increased immediately [Fig. 6(b)] and hemoglobins were oxygenated within $\sim 30 \mathrm{~s}$ [Fig. 6(c)] after starting oxygen supply. For this rat, $\mathrm{R}_{805}$ did not show a TSC-like change and $\mathrm{R}_{805}$ gradually recovered to its initial level with elapse of time [Fig. 6(a)]. $\mathrm{SpO}_{2}$ became $100 \%$ at $\sim 50 \mathrm{~s}$ and EEG activity recovered at $\sim 200 \mathrm{~s}$ after starting oxygen supply [Figs. 6(d) and 6(e)]. For both rats shown in
Figs. 7 and 8, reoxygenation was started during TSC, but one rat survived (Fig. 7) and the other rat died (Fig. 8). For the rat that survived (Fig. 7), $\mathrm{R}_{805}$ showed a scattering increase (phase I) at $\sim 140 \mathrm{~s}$ after starting $\mathrm{N}_{2}$ inhalation [Fig. 7(a)] and then reoxygenation was started in the following phase showing a scattering decrease (phase II). After starting oxygen supply, CBV was restored and hemoglobins were successfully oxygenated [Figs. 7(b) and 7(c)], during which time $\mathrm{R}_{805}$ showed a rapid decrease followed by a slight increase and then a further decrease to below the initial level and thereafter $\mathrm{R}_{805}$ recovered toward its initial level [Fig. 7(a)]. $\mathrm{SpO}_{2}$ and EEG activity also recovered, and the rat survived and functionally recovered. For the rat that died (Fig. 8), $\mathrm{R}_{805}$ showed a TSC after starting oxygen supply and, thereafter, it remained at a high level [Fig. 8(a)] as shown in Fig. 3(a). In this case, neither restoration of CBV nor hemoglobin oxygenation was observed [Figs. 8(b) and $8(\mathrm{c})$ ]. $\mathrm{SpO}_{2}$ could not be detected due to weakened arterial pulse and EEG activity was not recovered [Figs. 8(d) and 8(e)]. When rats were reoxygenated after TSC, temporal behaviors of signals were similar to those shown in Fig. 8 (data not shown).

In the present hypoxic brain model, for all rats that recovered with reoxygenation, regardless of reoxygenation timing, oxygen restoration to the brain was found to occur successfully and a TSC did not appear. For all rats that did not recover despite reoxygenation, on the other hand, a sign of oxygen restoration to the brain was not found and a TSC appeared. These results demonstrate that rat brains exposed to hypoxia can be saved if oxygen restoration to the brain can be made successfully until the end of TSCs. This is consistent with our speculation that cells in the brain can recover when oxygen is restored by phase II. However, we did not expect that cells in the brain would still be reversible even in phase III.

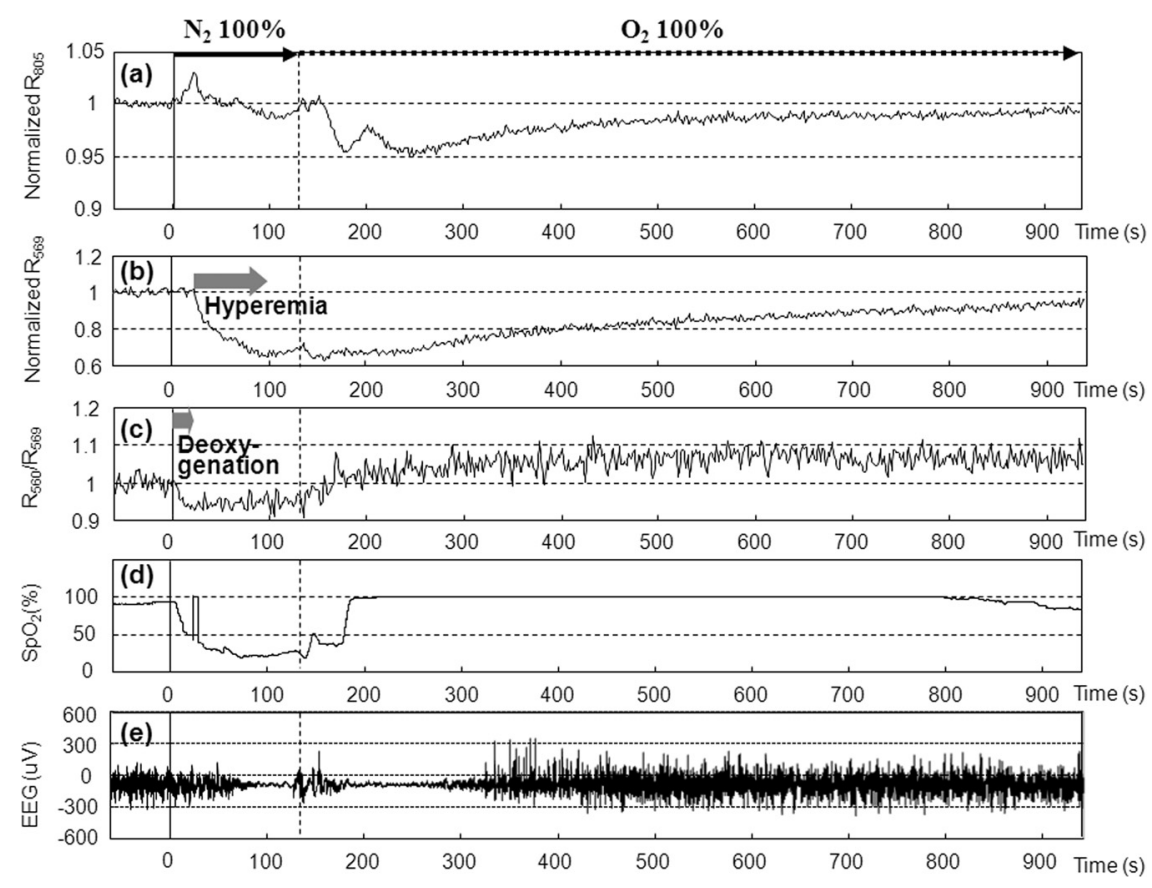

Fig. 6 Time courses of (a) normalized diffuse reflectance intensity at $805 \mathrm{~nm}$, (b) that at $569 \mathrm{~nm}\left(\mathrm{R}_{569}\right)$ indicating relative change in total $\mathrm{Hb}$ concentration, (c) that at $560 \mathrm{~nm}\left(\mathrm{R}_{560}\right)$ divided by $\mathrm{R}_{569}$, indicating relative change in deoxy-Hb concentration, (d) arterial oxygen saturation and (e) EEG activity. After starting nitrogen gas inhalation at $t=0$, oxygen gas supply was started at $t=130 \mathrm{~s}$ (dashed lines) before appearance of TSC. Reflectance intensity at $805 \mathrm{~nm}$ in (a) did not show a TSC. The rat survived and functionally recovered. 

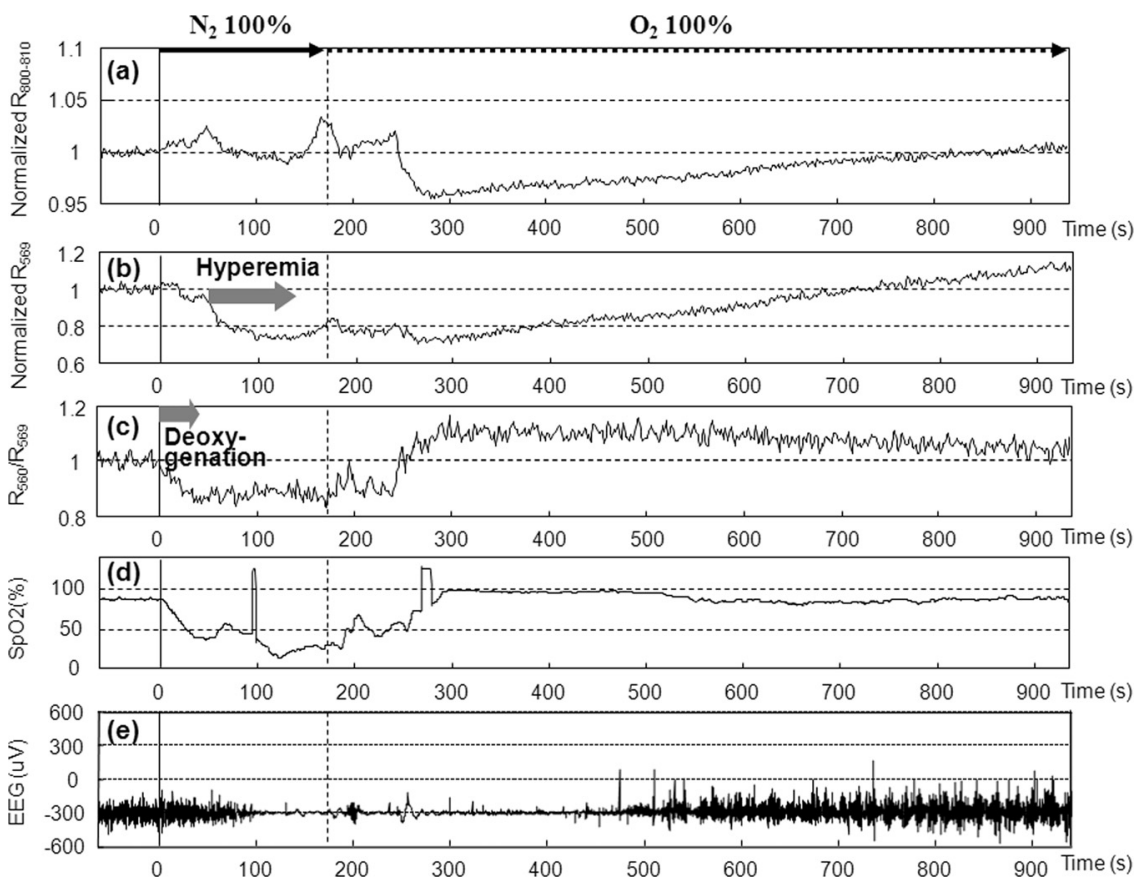

Fig. 7 Time courses of signals shown in Fig. 6. After starting nitrogen gas inhalation at $t=0$, the oxygen supply was started during phase II of the TSC expected. After starting oxygen gas supply, reflectance intensity at $805 \mathrm{~nm}$ in (a) showed a rapid decrease and then a further decrease to less than the initial level, and thereafter it recovered to its initial level. The rat survived and functionally recovered in this case.

The questions regarding the results shown in Fig. 5 are why survival rate is probabilistic in all phases of TSC and what determines survival or death of the rats during TSC. Here we provide possible answers to these questions. One factor determining the survival of rats during hypoxia would be associated with functional integrity of vasculatures in the brain when oxygen supply was started. As described above, TSC should reflect AD, and it is known that $\mathrm{AD}$ causes passive vascular collapse or vasoconstriction due to vasoconstrictive neurovascular coupling. ${ }^{16-18}$ Under the strong influence of these vascular responses, oxygen restoration to the brain tissue probably fails. Actually, spectral analysis of reflectance intensity changes can reveal the effect of vascular collapse or vasoconstriction; for example, a relatively large reflectance increase in the range of 500-600 nm
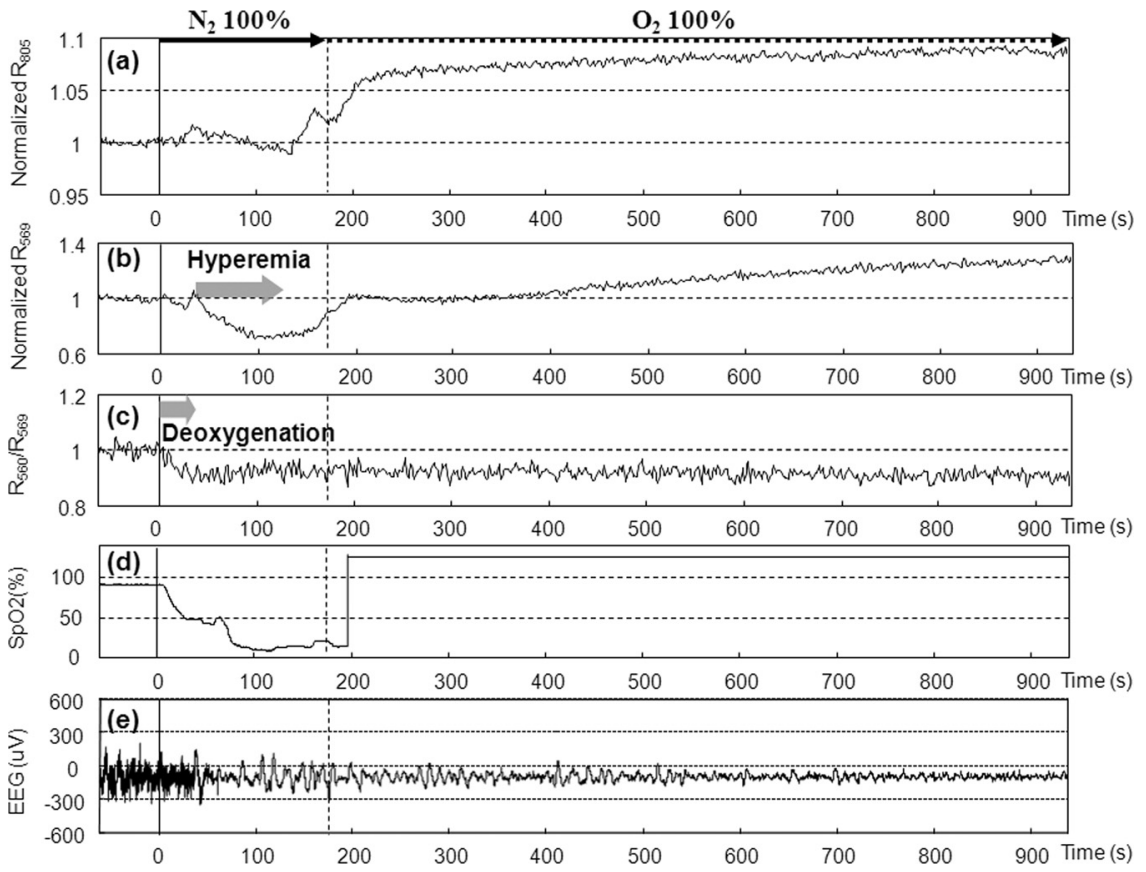

Fig. 8 Time courses of signals shown in Fig. 6. After starting nitrogen gas inhalation at $t=0$, oxygen gas supply was started during phase II of the TSC. In spite of the similar situation as that shown in Fig. 7, reflectance intensity at $805 \mathrm{~nm}$ showed TSC and the rat did not survive. 
[Figs. 4(b) and 4(c)] may indicate vascular collapse or vasoconstriction. Further study is needed to reveal the influence of neurovascular coupling on survival in the present rat model.

Another factor that should be considered to understand probabilistic survival is a limitation due to local measurement. Because AD is known to be initiated focally and to develop inhomogeneously in the cortex ${ }^{19-21}$ spatial behaviors of AD waves all over the brain should relate to survival of the rat. However, TSC was observed for a limited region of the brain in the present study, possibly causing an uncertainty to correlate optical signals with survival. Imaging of scattering signals throughout the brain would be useful to reduce this uncertainty. More inherently, oxygen restoration to the brain depends on systemic factors: respiration, circulation and others. ${ }^{22}$ An important part of our future study will be efforts to determine the correlations between scattering signals from local brain regions and these systemic factors.

Despite the remaining uncertain factors described above, the results of the present study indicate that the time span of TSC can be regarded as the critical time zone for survival of the present animal model; appropriate treatment conducted during TSC can rescue the rats in spite of having undergone severe hypoxic stress. In other words, light-scattering signal can be a good indicator of brain tissue viability. Because scattering signal measurement does not require data processing, the signal can be monitored in real time. Thus, by continuous monitoring of the light-scattering signal, we can know the start of TSC, which can be regarded as an alarm of entering a critical time zone. If we immediately start an intervention (e.g., blood reperfusion) in such a case, then brain tissue can be successfully rescued. After TSC, on the other hand, reperfusion would result in a catastrophic outcome due to intracranial hemorrhage; light-scattering signal may also be used to avoid such an adverse event. One of the ideal situations in which the scattering signal is used would be intraoperative monitoring of tissue viability during blood flow interruption by clamping and reperfusion. Continuous monitoring of the scattering signal would also be useful in critical care medicine. Optical absorption signals related to hemodynamics can also be simply measured in real time. Simultaneous measurements of the scattering and absorption signals, as well as conventional systemic biological signals, can further increase the usefulness of scattering signals.

\section{Conclusions}

In the present study, we performed multiwavelength diffuse reflectance measurements for rat brains during hypoxia caused by $\mathrm{N}_{2}$ inhalation under spontaneous respiration and demonstrated that TSC, which was observed in our previous bloodfree rat brain model, can be detected even in the presence of blood in vivo by using NIR light. Experiments to reoxygenate rats showed that their recovery rates depended on the timing of oxygen supply: $100 \%$ recovery by oxygen supply starting before TSC and $0 \%$ recovery by oxygen supply starting after TSC. When oxygen was supplied to rats during TSC, survival was probabilistic and its TSC phase dependence was not clear. For the rats that survived, disability of motor function was not observed. The reason for the probabilistic survival is not clear, but it might be associated with vascular response to anoxic depolarization in the brain and systemic respiratory and circulatory disturbance under the condition of severe hypoxic stress. The time span showing TSC can be regarded as the critical time zone for cardiopulmonary-cerebral resuscitation in the present severe hypoxic model. These findings indicate that the light-scattering signal can be used as an indicator of reversibility of brain tissue, providing useful information on the critical time zone for treatment to rescue the brain exposed to severe hypoxia.

\section{Acknowledgments}

The authors gratefully acknowledge the financial support of the Kowa Life Science Foundation. We thank Dr. Hiroaki Kobayashi for technical assistance in surgical procedures in animal experiments.

\section{References}

1. K. A. Hossmann, "Viability thresholds and the penumbra of focal ischemia," Ann. Neurol. 36, 557-565 (1994).

2. J. R. Mourant and I. J. Bigio, "Elastic-scattering spectroscopy and diffuse reflectance," Chapter 29 in Biomedical Photonics Handbook, T. Vo-Dinh, Eds., pp. 29-1-29-5, CRC Press, Boca Raton (2003).

3. P. Van Der Zee, M. Essenpreis, and D. T. Delpy, "Optical properties of brain tissue," Proc. SPIE 1888, 454-465 (1993).

4. D. L. Nelson and M. M. Cox, "Oxidative phosphorylation and photophosphorylation," in Lehninger Principles of Biochemistry, D. L. Nelson and M. M. Cox, Eds., pp. 659-673, Worth Publishers, New York (2000).

5. S. Kawauchi, S. Sato, H. Ooigawa, H. Nawashiro, M. Ishihara, and M. Kikuchi, "Simultaneous measurement of changes in light absorption due to the reduction of cytochrome c oxidase and light scattering in rat brains during loss of tissue viability," Appl. Opt. 47(22), 4164-4176 (2008).

6. S. Kawauchi, S. Sato, H. Ooigawa, H. Nawashiro, M. Ishihara, and M. Kikuchi, "Light scattering change precedes loss of cerebral adenosine triphosphate in a rat global ischemic brain model," Neurosci. Lett. 459(3), 152-156 (2009).

7. A. J. Hansen, "Effect of anoxia on ion distribution in the brain," Physiol. Rev. 65, 101-148 (1985).

8. G. G. Somjen, "Mechanisms of spreading depression and hypoxic spreading depression-like depolarization," Physiol. Rev. 81, 1065-1096 (2001).

9. I. Joshi and R. D. Andrew, "Imaging anoxic depolarization during ischemia-like conditions in the mouse hemi-brain slice," J. Neurophysiol. 85(1), 414-424 (2001).

10. L. Tao, D. Masri, S. Hrabetova, and C. Nicholson, "Light scattering in rat neocortical slices differs during spreading depression and ischemia," Brain Res. 952, 290-300 (2002).

11. M. Balestrino, "Pathophysiology of anoxic depolarization: new findings and a working hypothesis," J. Neurosci. Methods 59(1), 99-103 (1995).

12. S. Prahl, "Optical Absorption of Hemoglobin," Oregon Medical Laser Center (1999), http://omlc.ogi.edu/spectra/hemoglobin/, last accessed Oct. 10, 2010

13. P. Van Der Zee, "Measurement and modelling of the optical properties of human tissue in the near infrared," $\mathrm{PhD}$ Thesis, University College London, pp. 266-269 (1992).

14. M. Firbank, M. Hiraoka, M. Essenpreis, and D. T. Delpy, "Measurement of the optical properties of the skull in the wavelength range 650-950 nm," Phys. Med. Biol. 38, 503-510 (1993).

15. T. Takano, G. F. Tian, W. Peng, N. Lou, D. Lovatt, A. J. Hansen, K. A. Kasischke, and M. Nedergaard, "Cortical spreading depression causes and coincides with tissue hypoxia," Nat. Neurosci. 10, 754-762 (2007).

16. K. A. Hossmann, "Reperfusion of the brain after global ischemia: hemodynamic disturbances," Shock 8(2), 95-101 (1997). 
17. H. K. Shin, A. K. Dunn, P. B. Jones, D. A. Boas, M. A. Moskowitz, and C. Ayata, "Vasoconstrictive neurovascular coupling during focal ischemic depolarizations," J. Cereb. Blood Flow Metab. 26(8), 10181030 (2006).

18. P. B. Jones, H. K. Shin, D. A. Boas, B. T. Hyman, M. A. Moskowitz, C. Ayata, and A. K. Dunn, "Simultaneous multispectral reflectance imaging and laser speckle flowmetry of cerebral blood flow and oxygen metabolism in focal cerebral ischemia," J. Biomed. Opt. 13(4), 044007 (2008).

19. A. A. P. Leao, "Further observations on the spreading depression of activity in the cerebral cortex," J. Neurophysiol. 10, 409-414 (1947).
20. P. G. Aitken, G. C. Tombaugh, D. A. Turner, and G. G. Somjen, "Similar propagation of SD and hypoxic SD-like depolarization in rat hippocampus recorded optically and electrically," J. Neurophysiol. 80, 1514-1521 (1998).

21. C. R. Jarvis, T. R. Anderson, and R. D. Andrew, "Anoxic depolarization mediates acute damage independent of glutamate in neocortical brain slices," Cereb. Cortex 11, 249-259 (2001).

22. H. H. Hendrickx, G. R. Rao, P. Safar, and S. E. Gisvold, "Asphyxia, cardiac arrest and resuscitation in rats. I. Short term recovery," Resuscitation 12(2), 97-116 (1984). 